\title{
Prevalencia y factores asociados a la presencia de reacciones adversas a medicamentos en los pacientes tratados por medicina interna en el Hospital Universitario Hernando Moncaleano Perdomo
}

\author{
Carmen Rosa Rangel ${ }^{1}$, Silvia Rojas ${ }^{1}$, Mileidys López ${ }^{1}$, Giovanni Caviedes ${ }^{2}$. \\ Médico interno Hospital Universitario Hernando Moncaleano Perdomo. Universidad de Sucre \\ Medico Internista, Farmacólogo clínico, Epidemiólogo. Docente, Universidad Surcolombiana.
}

Fecha de recepción: Noviembre 15 de 2015

Fecha de corrección: Febrero 23 de 2016

Fecha de aprobación: Marzo 13 de 2016

\section{Resumen}

Objetivo: Estimar la prevalencia y factores asociados para presentar reacciones adversas a medicamentos en los pacientes tratados por medicina interna del Hospital Universitario Hernando Moncaleano Perdomo (HUHMP) durante un período de 2 meses. Materiales y métodos: Estudio prospectivo de corte transversal. El análisis estadístico se realizó con Epi info v7.0 Resultados: De los 284 pacientes, se obtuvieron 89 reacciones adversas a medicamentos (RAM) que corresponde al $31.34 \%$ de la muestra, el $52.4 \%$ eran del género masculino con una edad media de 58.5 años. Las RAM se clasificaron según su mecanismo de producción en tipo A en $79 \%$, tipo B $14 \%$ y tipo C $5 \%$; la mayoría fueron leves en el $88 \%$ de los casos. El principal sistema farmacológico afectado fue el gastrointestinal seguido por el hematológico en un $22 \%$ y $19 \%$ respectivamente; la enoxaparina fue el principal fármaco relacionado con la producción de RAM en el 17\%; al igual es el fármaco con más interacciones medicamentosas junto con el ácido acetilsalicílico en el 26\% de los casos. Entre los factores asociados para la producción de RAM son la polifarmacia IC: 1.965.57, p 0.000027 y las interacciones medicamentosas IC: $1.32-3.81, p 0.0011$. Conclusiones: Las interacciones medicamentosas y la polifarmacia son los factores asociados a la presencia de reacciones adversas a medicamentos en pacientes de medicina interna.

Palabras Clave: Anomalías Inducidas por Medicamentos, Combinación de Medicamentos, Interacciones de Drogas,
Prevalence and risk factors related with adverse reactions to drugs in patients from Hospital Universitario Hernando Moncaleano Perdomo

\begin{abstract}
Objective: estimate the prevalence and the associated factors to presenting adverse reactions for medicines in patients treated for internal medicine of the Hernando Moncaleano Perdomo university hospital (HMPUH) for two months. Methods: prospective study of transversal cut. Statistical analysis was carried it out with Epi info v7.0. Results: from the 284 patients, it obtained 89 adverse reactions for medicines (ADR) that come from to $31.34 \%$ of sample, the $52,4 \%$ were male with an average age of 58,5 years old. The ADR were classified according to their production mechanism in A sort $79 \%$, B sort $14 \%$ and C sort $5 \%$; the majority were minor in the $88 \%$ of cases. The main pharmacological system affected was the gastrointestinal followed by the hematological in $22 \%$ and $19 \%$ respectively; the enoxaparin was the main drug associated with the ADR production in the $17 \%$, so is the drug with more drug interactions together to the acetylsalicylic acid in the $26 \%$ of the cases. Among the associated factors for the ADR production are the polypharmacy IC: $1.96-5.57, \mathrm{p} 0.000027$ and the drug interactions IC: $1.32-3.81$, p 0.0011 . Conclusions: the drug interactions and the polypharmacy are the associated factors to the presence of adverse reactions to medicines in internal medicine patients.

Keywords: Abnormalities, Drug-Induced, Drug Combinations, Drug Interactions
\end{abstract}

\section{Introducción}

Las reacciones adversas a medicamentos (RAM) constituyen un problema de salud pública. En un meta-analisis realizado por Bouvy y cols. El porcentaje de admisiones a los centro de urgencias por reacciones adversas a medicamentos fue de $3.5 \%$ y un $10.1 \%$ de los pacientes presenta alguna RAM durante su estancia hospitalaria ${ }^{1}$. también se ha reportado que alrededor del $5 \%$ de todas las hospitalizaciones se atribuye a algún tipo de reacción adversa, y el $20 \%$ de los pacientes hospitalizados pueden presentar las RAM ${ }^{2}$. Estos eventos disminuyen la calidad de vida de los enfermos, prolonga la estancia hospitalizaría, aumenta la morbi-mortalidad, ocasiona perdida de la confianza en los médicos y eleva el costo de la atención médica. Los resultados de los estudios clínicos realizados antes de la comercialización, tienen ciertas limitaciones en cuanto al número de personas expuestas a medicamentos, tiempo de exposición al mismo y la generalización de los resultados a la población en general.

Para este estudio se denomina interacción medicamentosa a toda identificación que se pueda producir sobre la farmacocinética o farmacodinamia del medicamento, debido a la interacción con compuestos endógenos o exógenos como ocurre en la administración simultanea de otros fármacos y demás factores como alimentos, bebidas o contaminantes ambientales ${ }^{3}$.

Existen muchos factores de riesgo para el desarrollo de reacciones adversas a medicamentos entre los cuales se encuentran la polifarmacia y las alteraciones farmacocinéticas propias de los pacientes críticos. Sin embargo la cultura del reporte de las reacciones adversas no está bien establecida en muchos sitios, por lo cual hay pocos reportes de estos eventos en pacientes hospitalizados y ambulatorios ${ }^{2}$. Jason Lazarou y colaboradores en un meta-análisis de la incidencia de las reacciones adversas a medicamentos en los pacientes hospitalizados encontraron factores de riesgo asociados a la edad, el tiempo de estancia prolongado, el género femenino y la polifarmacia. La incidencia de reacciones adversas fue de $2.1 \%$ y de reacción adversa fatal fue $0.19 \%$. Así mismo la incidencia de RAM no ha cambiado a través de tres décadas ${ }^{4}$.

Daniel E.Becker y colaboradores mostraron que las interacciones potenciales usualmente son más probables que las interacciones reales, partiendo de la variabilidad de cada individuo ante cada medicamento. Se estima que alrededor del $10 \%$ de las personas hospitalizadas han estado en alerta de interacciones medicamentosa, mientras aproximadamente el $25 \%$ han tenido un efecto adverso a un medicamento ${ }^{5}$. 
En la región Surcolombiana se encuentra publicado un estudio sobre reacciones adversas a medicamentos en el servicio de medicina interna del Hospital Universitario por Beltrán y cols. Ellos reportan un estudio descriptivo de corte transversal prospectivo, con un total de 303 pacientes, de los cuales el 50\% que corresponden a 153 pacientes presentaron algún tipo de reacción adversa, el 63,4\% correspondían al servicio de urgencias; el 57,5\% de los casos fueron hombres y el $66 \%$ provenían de área urbana. La media de la edad fue $63+/-4,6$. Las RAM presentadas por los pacientes, se agruparon por sistemas; de estas el 27,4\% corresponde a piel, seguido del gastrointestinal con un $13,8 \%$, del sistema hematológico y cardíaco en un $13,2 \%$ cada uno ${ }^{6}$.

En el presente estudio se profundizará en los factores asociados que contribuyen a presentar una RAM, incluyendo las interacciones medicamentosas, compromiso renal y hepático en los pacientes que fueron admitidos en el Hospital Univesitario Hernando Moncaleano Perdomo para ser tratados por la especialidad de medicina Interna.

\section{Materiales y Métodos}

Se realizó un estudio transversal. Se calculó el tamaño de la muestra:

Donde n: es el tamaño de la muestra; $\mathrm{Z}$ es una constante que denota el valor de la desviación normal, para un nivel de significación alfa igual a 5\%; P es la proporción estimada del evento; Q es igual a 1-P; y E es equivalente a la precisión, que hace referencia a cuanto se aleja la muestra del verdadero porcentaje del universo. Con base en estos parámetros se una precisión del $5 \%$, una proporción de evento de $25 \%$ (Dato adquirido de lo descrito en la literatura ${ }^{7}$ ), en una población de 19.413 que fueron el total de pacientes admitidos en un año en el Hospital, para ser tratados por el servicio de medicina interna, se obtuvo una muestra de 284 pacientes. Como

\section{Criterios de inclusión:}

1. Todos los pacientes que presentaron signos, síntomas y estudios de laboratorios compatibles con RAM,

2. Pacientes hospitalizados en el servicio de Medicina interna piso y urgencias; como criterio de exclusión los pacientes hospitalizados en Unidad de cuidados intensivos.

Las variables se recolectaron por medio observación directa, se realizó una encuesta en la que los investigadores interrogaban a cada paciente por signos y síntomas, además se revisaban las historias clínicas para complementar las información de las variables que contaban con características demográficas, factores relacionados con el paciente como el diagnostico principal por el que estaba hospitalizado, comorbilidades, estado nutricional, compromiso renal y hepático. También la presencia de reacciones adversas, y si éstas estaban presentes eran clasificadas dependiendo su mecanismo de producción, severidad, causalidad por medio del algoritmo de Naranjo y las interacciones con otros medicamentos. Para en análisis de las interacciones, se ingresaron los medicamentos administrados a los pacientes en un programa virtual llamado Medscape drugs interaction checker.

Para el análisis estadístico los datos se tabularon en una hoja Excel 2013 y se analizaron los resultados con el software Epi info v7.0. Se realizó una estadística descriptiva con la obtención de medidas de tendencia central y de dispersión.

Se solicitó previo consentimiento al comité de ética del HUHMP por tratarse de un estudio de revisión de historias clínicas. Aunque la información obtenida de los pacientes, familiares e historias clínicas para la elaboración de este trabajo se realizó única y exclusivamente con fines científicos e investigativos, respetando de ésta manera el derecho de reserva de identidad y manteniendo la integridad de los pacientes. Este estudio es considerado de bajo riesgo.

\section{Resultados}

Cuadro 1 características demográficas y clínicas de los pacientes evaluados

\begin{tabular}{ll}
\hline \multicolumn{1}{c}{ Variable } & \multicolumn{1}{c}{ Resultado } \\
\hline Género, n(\%) & $149(52.47)$ \\
Masculino & $135(47.54)$ \\
Femenino & $58.5(38.5-96)$ \\
Edad, mediana(RI) & \\
Procedencia, n(\%) & $179(63.03)$ \\
Urbano & $105(36.97)$ \\
Rural & $5(3.5-16)$ \\
Número de Medicamentos, media(RI) & \\
Nutrición, n(\%) & $274(96.48)$ \\
Normal & $10(3.52)$ \\
Enteral & 0 \\
Parenteral & \\
Compromiso Renal, n(\%) & $236(83.09)$ \\
No & $48(16.9)$ \\
Si & \\
Compromiso hepático, n(\%) & $30(10.56)$ \\
Si & $254(89.44)$ \\
No &
\end{tabular}

De 284 pacientes ingresaron en un año al servicio de urgencias para ser tratados por medicina interna. Las edades se reportaron entre 15 a 96 años, con una mediana de 58.5 años; el 63\% de todas las personas evaluadas provenían de área urbana; en promedio los pacientes eran tratados con 5 medicamentos; el mayor porcentaje alimentados por vía normal en un $96 \%$, al momento del ingreso presentaron compromiso renal o compromiso hepático el $16.9 \%$ y $10.5 \%$ respectivamente (Cuadro 1).

Cuadro 2 Diagnósticos de los pacientes evaluados

\begin{tabular}{lc}
\hline \multicolumn{1}{c}{ Diagnóstico } & Resultado, n (\%) \\
\hline Enfermedad pulmonar obstructiva crónica & $16(5.63)$ \\
Síndrome Febril & $14(4.93)$ \\
Dengue con signos de alarma & $14(4.93)$ \\
Falla cardiaca & $13(4.57)$ \\
Infección de vías urinarias & $12(4.23)$ \\
Cefalea & $9(3.17)$ \\
Dolor Torácico & $8(2.82)$ \\
Hemorragia de vías digestivas altas & $8(2.82)$ \\
Pie diabético & $7(2.46)$ \\
Pancitopenia & $7(2.46)$ \\
Neumonía & $7(2.46)$ \\
Diarrea & $7(2.46)$ \\
Otros & $162(57.04)$ \\
& $\mathbf{2 8 4}(\mathbf{1 0 0})$ \\
\hline
\end{tabular}

Como diagnóstico más frecuente fue la exacerbación de enfermedad pulmonar obstructiva crónica en un 5.6\%, seguido por síndrome 
febril en estudio y dengue con signos de alarma en un $4.93 \%$ en ambas patologías, falla cardiaca en un $4.57 \%$, infección de vías urinarias en un $4.23 \%$, cefalea en un $3.17 \%$ entre otros (Cuadro 2).

De los 284 pacientes, el 31.34\% presentaron RAM que se le estaban administrando; al clasificar las reacciones adversas según su mecanismo generador se encontró que el 79\% correspondían al tipo A, el $13 \%$ al tipo B, y en menor proporción en un $5 \%$ al tipo C. De acuerdo a su causalidad aplicando el algoritmo de Naranjo podemos decir que el $66 \%$ de las RAM fueron definitivas, el $21 \%$ probables y en igual proporción dudosas y no relacionadas en un $1 \%$ de los casos. La severidad de las RAM se clasificó de leve a grave, con resultados que mostraron que el $88 \%$ eras leves, el $9 \%$ moderadas y el $2 \%$ graves. Cuadro 3 Medicamentos asociados a las reacciones adversas, sistema involucrado, vía y tiempo de exposición del fármaco

\begin{tabular}{lc}
\hline \multicolumn{1}{c}{ Variable } & Resultado, $\mathrm{n}(\%)$ \\
Medicamento & \\
Enoxaparina & $17(17,98)$ \\
Omeprazol & $13(14.61)$ \\
Salbutamol & $3(3.37)$ \\
Tramadol & $3(3.37)$ \\
Trazodona & $3(3.37)$ \\
Losartan & $3(3.37)$ \\
Otros & $47(52.80)$ \\
Sistema Farmacológico & \\
Gastrointestinal & $20(22.73)$ \\
Hematológico & $17(19.32)$ \\
Infeccioso & $13(14,77)$ \\
Neurológico & $14(15.91)$ \\
Cardiovascular & $9(10.23)$ \\
Respiratorio & $5(5.68)$ \\
Otros & $11(12.35)$ \\
Días de exposición & \\
0 - 1 día & $4(4.49$ \\
2 - 3 días & $44(49.44)$ \\
4 - 5 días & $21(23.60)$ \\
- 10 días & $9(10.11)$ \\
Mayor a 15 días & $11(12.36$ \\
Intrave de administración & \\
Inhalado & $22(24.72)$ \\
Oral & $3(3.37)$ \\
Subcutánea & $44(49.44)$ \\
Tópica & $19(21.35)$ \\
\hline & $1(1.12)$ \\
\hline
\end{tabular}

Con respecto a los medicamentos relacionados con las reacciones adversas, la enoxaparina fue la que más causó RAM en el 17\% de los casos, seguido por el omeprazol 14\%, salbutamol, tramadol trazodona y losartán con igual frecuencia en un 3.37\%. Las RAM presentadas por los pacientes se agruparon en sistemas farmacológicos; de estas el $22 \%$ afectaron el sistema gastrointestinal, hematológico en $19 \%$, infeccioso el 14\%; las RAM empezaron a producirse en los primeros 2 días en el $49.4 \%$ de los pacientes y la principal vía de administración de estos medicamentos fue la oral en el $49 \%$ de los casos seguida por la intravenosa en un 24\% (Cuadro 3).
Cuadro 4 Frecuencia de interacciones medicamentos y clasificación

\begin{tabular}{lll}
\hline $\begin{array}{c}\text { Interacciones medicamen- } \\
\text { tosas }\end{array}$ & $\mathbf{( \% )}$ & \multicolumn{1}{c}{$\begin{array}{c}\text { Clasifica- } \\
\text { ción ATC* }\end{array}$} \\
Enoxaparina - ASA & 26.7 & $\mathrm{C}+\mathrm{B}$ \\
Enalapril - ASA & 8.92 & $\mathrm{C}+\mathrm{B}$ \\
Omeprazol - Ampicilina & 5.35 & $\mathrm{~A}+\mathrm{J}$ \\
Omeprazol - Losartan & 5.35 & $\mathrm{~A}+\mathrm{C}$ \\
Enoxaparina - Acetaminofén & 3.57 & $\mathrm{~B}+\mathrm{V}$ \\
Enoxaparina - Enalapril & 3.57 & $\mathrm{~B}+\mathrm{C}$ \\
Otros & 46.42 & \\
\hline
\end{tabular}

Se encontraron interacciones medicamentosas relacionadas con los fármacos administrados a los pacientes con RAM, entre ellas la más frecuente fue la asociada con enoxaparina y ácido acetilsalicílico en el 26\% de los casos con un tipo de interacción significativa, los cuales actúan en el sistema hematológico y cardiovascular respectivamente (Cuadro 4).

Cuadro 5. Modelo multivariado para el ajuste de confusores

\begin{tabular}{ccccc}
\hline Factor asociado & OR & IC & $\mathbf{P}$ & $\mathbf{C h i}^{\mathbf{2}}$ \\
Género & 0.83 & $(0.50-1.38)$ & 0.24 & 0.47 \\
Procedencia & 0.92 & $(0.55-1.55)$ & 0.38 & 0.08 \\
Polifarmacia & 3.30 & $(1.96-5.57)$ & 0.000027 & 21.09 \\
Compromiso renal & 1.24 & $(0.65-2.4)$ & 0.25 & 0.44 \\
Compromiso hepático & 1.25 & $(0.70-3.33)$ & 0.14 & 1.16 \\
Interacciones & 2.24 & $(1.32-3.81)$ & 0.0011 & 9.21 \\
medicamentosas & & &
\end{tabular}

Se hizo un análisis de confusores utilizando como variable dependiente la presencia de reacciones adversas a medicamentos y como variables independientes la procedencia, genero, polifarmacia, compromiso renal y/o hepático y las interacciones con otros medicamentos, dando como significancia estadística, la asociación de polifarmacia $I C_{95 \%}$ : 1,96-5.57, para un valor de p 0,000027 y las interacciones medicamentosas $I C_{95 \%}: 1.32$ - 3.81, y un valor de p 0,0011 (Cuadro 5). Estos valores están ajustados por género, procedencia de los pacientes, compromiso renal y hepático.

\section{Discusión}

Una de las principales motivaciones en la realización de éste estudio, es sin duda que muchos de los paciente manejados por el servicio de medicina interna requieren ser polimedicados por sus comorbilidades. La comprensión de ésta situación sumando otros factores como el compromiso renal o hepático, además de las interacciones medicamentosas nos permite como personal de la salud, preveer e inclusive evitar en algunos casos las RAM.

En el estudio de la Dra. Pedrós y colaboradores titulado Prevalencia, factores de riesgo y principales características de las reacciones adversas a los medicamentos que conducen a la hospitalización ${ }^{8}$, el cual se realizó durante un periodo de 120 días, encontrándose que la polifarmacia y la edad eran los principales factores de riesgo para desarrollar reacciones adversas, lo cual se incrementaba con el número de medicamentos que usaban al momento de la admisión al hospital: 3-5 drogas con un OR 5.07 (IC 95\% 2.71 - 9.59), 6-9 drogas OR 5.90 (IC 95\% 3.16- 11.96), > 10 drogas OR 8.94 (IC 95\% 4.7316.89). Al compararse estos resultados con nuestra investigación, se encuentra resultados similares; en nuestro estudio un probable exceso de riesgo para presentar RAM fue la polifarmacia ( $>5$ medicamentos) 
con un OR 3.30 IC 95\% 1.96-5.57 cuando se ajustaron varios confusores. Lo anterior puede estar explicado por el efecto sinérgico en cuanto a toxicidad que se obtiene con algunas combinaciones de fármacos, siendo mayor el riesgo cuando se utiliza un agente. ${ }^{9}$ De aquí la importancia de racionalizar el uso de medicamentos, y con esto disminuir la prevalencia de posibles efectos adversos.

Otro aspecto a considerar es el incremento de la sensibilidad farmacodinámica y farmacocinética dado por el compromiso renal y/o hepático con la aparición de RAM. Muchos son los estudios que han demostrado una clara y significativa asociación entre insuficiencia renal y aparición de eventos adversos ${ }^{10}{ }^{11}$, como por ejemplo en estudio ralizado por el Dr. Yen-Chia Chen et $\mathrm{All}^{10}$, en el cual se encontraba asociación cuando los valores de creatinina sérica eran mayor 1.4 [IC 95\% $1.8(1.3-2.5)$ ], explicándose por la disminución en el aclaramiento del medicamento y sus metabolitos. Sin embargo en este mismo estudio sugieren ser cauteloso con esta asociación, ya que la interacción fármaco-enfermedad o fármaco-fármaco pueden jugar un papel más importante en el aumento del riesgo de RAMs más que la elevación de creatinina sérica. Siendo esto consistente con nuestro estudio, en el cual si se encontró relación de la alteración renal y RAMs [OR 1.24 (IC 95\% 0.65-2.4)], aunque su fuerza de asociación fue inferior al probable exceso de riesgo comparado con el de las interacciones medicamentosas; lo mismo se encontró en relación a la alteración de la función hepática. Sin embargo no hay que pasar por el alto el ajuste de las dosis de medicamentos de acuerdo a la función renal, en especial en edades avanzadas, con el fin de prevenir ese tipo de RAMs.

En el estudio por Beltrán y cols. Las interacciones farmacocinéticas se presentaron en un $27.2 \%$ de los pacientes y las más frecuentes fueron las producidas a nivel del metabolismo con el $61.3 \%$ las cuales implican las vías metabólicas del citocromo $\mathrm{p} 450$ por el uso conjunto de fármacos que la inhiban o la induzcan a nivel nuclear. El ácido acetil salicílico fue el fármaco que más interacciona con otros medicamentos; con la enoxaparina en un $7.12 \%$, con el enalapril en un 5.6\%, con el metropolol en un 3.5\%, con el losartán en un $2.8 \%$ y con la furosemida en un $2.2 \%{ }^{6}$. En nuestro estudio encontramos un resultado similar ya que la interacción de enoxaparina - ácido acetil salicílico se presentó en un $26 \%$ y fue la más frecuente.

En un estudio de casos y controles realizado por Medina et al, titulado Factores asociados con la presentación de reacciones adversas a medicamentos en pacientes que acuden al servicio de urgencia de un hospital general de Granada España los órganos y sistemas más afectados por las RAM fueron: sistema digestivo $(41,8 \%)$, piel $(31,6 \%)$, sistema nervioso $(11,9 \%)$ y cardiovascular $(11 \%)^{12}$ lo cual comparado con nuestro estudio coincide en que el sistema más afectado fue el gastrointestinal con un $22 \%$.

En el mismo estudio del hospital de Granada se presentó que un $68,7 \%$ de las RAM fueron del tipo A y el 31,3\% restante del tipo $\mathrm{B}^{12}$. En nuestro estudio el $71 \%$ correspondieron a RAM tipo A, un $13 \%$ a tipo B y el $5 \%$ restante a tipo C.

Se encontró que existen múltiples factores asociados a la presencia de RAM en los pacientes manejados por medicina interna, donde la polifarmacia es uno de los principales, además del número de interacciones medicamentosas, el compromiso renal y hepático. Las RAM más frecuentes fueron las tipo A y leves, resultando como principales medicamentos causantes la enoxaparina y el omeprazol, afectado el aparato gastrointestinal seguido del hematológico. Se sugiere que el sistema de vigilancia de reacciones medicamentosas definido por este estudio se implemente particularmente en pacientes que tienen más de 5 fármacos ya que en ellos parece mas probable la aparición de efectos adversos asociados a medicamentos.

\section{Referencias}

1. Jacoline C. Bouvy, Marie L. De Bruin, Marc A. Koopmanschap. Epidemiology of adverse drug reactions in Europe: A review of recent observacional studies. Drug Saf. 2015; 38(5): 437-453.

2. Cervantes EI. Farmacovigilancia en cuidado intensivo. En: Gutierrez P, Carrillo E, editores. Guía farmacológica en la UCI adultos. México: Mc Graw Hill 2007; p. 26-27.

3. Osterhoudt KC, Pening TM. Toxicidad e intoxicaciones por fármacos. En: Goodman y Gilman. Las bases farmacológicas de la terapéutica. Mc Graw Hill. 12 edición. 2011, p 73-87

4. Lazarou J, Bruce H, Corey Paul N. Incidence of adverse drug reactions in hospitalized patients. JAMA, 1998; 279:1200-1205.

5. Daniel E. Becker. Pharmacodynamic Considerations for Moderate and Deep Sedation. Anesthesia Progress: Spring 2012, 59 (1): 2842

6. Cindy Lorena Beltrán Endo, Giovanni Caviedes Perez, Adverse drug reactions occurring in the internal medicine service at university hospital in Neiva. 2013. RFS p (26-31)

7. Jankel F, Saha L, Pandhi P M a lh o t ra S. Adverse Drug Event (ADE) related Medical Emergency D epartment visits and hospital admissions: a prospective study f rom a North Indian Referral Hospital. Journal of Clinical and Diagnostic Research 2008;2(1):600-604.

8. Consuelo Pedrós, Beatriz Quintana, Et al. Prevalence, risk factors and main features of adverse drug reactions leading to hospital admission. European Journal of Clinical Pharmacology. March 2014, Volume 70, Issue 3, pp 361-367. doi:10.1007/s00228-013$1630-5$

9. Routledge P A, O’Mahony M S, Woodhouse K W. Adverse drug reactions in elderly patients. Br J Clin Pharmacol. 2004 Feb; 57(2): 121-126. doi: 10.1046/j.1365-2125.2003.01875.x

10. Yen-Chia Chen, Ju-Sing Fan, Et al. Risk factors associated with adverse drug events among older adults in emergency department. European Journal of Internal Medicine 25 (2014) 49-55.

11. Anders Hellde'n,1 Ulf Bergman, Et al. Adverse Drug Reactions and Impaired Renal Function in Elderly Patients Admitted to the Emergency Department. Drugs Aging 2009; 26 (7): 595-6061170229X/09/0007-0595/\$49.95/0.

12. 12. Medinaa, E. Puchea y J. de Dios Lunab. Factores asociados con la presentación de reacciones adversas a medicamentos en pacientes que acuden al servicio de urgencia de un hospital general: estudio de casos y controles M.A. Junio 2000 